\title{
Drug-Induced Inhibition of Mitochondrial Fatty Acid Oxidation and Steatosis
}

\author{
Julie Massart • Karima Begriche • Nelly Buron • \\ Mathieu Porceddu • Annie Borgne-Sanchez • \\ Bernard Fromenty
}

Published online: 28 June 2013

(C) Springer Science+Business Media New York 2013

\begin{abstract}
Drug-induced inhibition of mitochondrial fatty acid $\beta$-oxidation (mtFAO) is a key mechanism whereby drugs can induce steatosis. The type and severity of this liver lesion is dependent on the residual mtFAO flux. Indeed, a severe inhibition of mtFAO leads to microvesicular steatosis, hypoglycemia and liver failure, which can be favored by genetic predispositions. In contrast, moderate impairment of mtFAO can cause macrovacuolar steatosis, which is by itself a benign lesion. In the long-term, however, macrovacuolar steatosis can progress with some drugs to steatohepatitis. Interestingly, drugs that are more likely to cause steatohepatitis are those impairing the mitochondrial respiratory chain (MRC) activity. Indeed, MRC impairment favors not only hepatic fat accretion but also oxidative stress and lipid peroxidation. Drugs inhibiting mtFAO could be more toxic in obese patients with preexisting nonalcoholic fatty liver disease (NAFLD) since higher mtFAO is a key metabolic adaptation to curb fat accretion during NAFLD.
\end{abstract}

Keywords Drug - Fatty acid oxidation - Fatty liver . Mitochondria $\cdot$ Respiratory chain $\cdot$ Steatosis

\footnotetext{
J. Massart

Department of Molecular Medicine and Surgery, Karolinska Institutet, Karolinska University Hospital, 17177 Stockholm, Sweden

K. Begriche · B. Fromenty $(\bowtie)$

INSERM, U991, Université de Rennes 1, 2, Avenue du Professeur Léon Bernard, 35043 Rennes Cedex, France e-mail: bernard.fromenty@inserm.fr

N. Buron · M. Porceddu - A. Borgne-Sanchez Mitologics SAS, Hôpital Robert Debré, 48 Boulevard Sérurier, 75019 Paris, France
}

\section{Introduction}

Drug-induced liver injury (DILI) includes several kinds of lesions such as acute hepatitis, cholestasis, phospholipidosis and steatosis $[1 \bullet \bullet, 2]$. This latter lesion corresponds to accretion of lipids, mainly triglycerides, although other lipids species can also accumulate such as free fatty acids (FAs) and acyl-carnitine derivatives [3, 4]. Actually, drugs are able to induce either microvesicular steatosis or macrovacuolar steatosis, and sometimes both kinds of lipid deposition [3, 5]. In this review, we will discuss how drugs can induce hepatic steatosis by disturbing the mitochondrial fatty acid oxidation (mtFAO) pathway [3-7]. However, before considering drug-induced hepatic steatosis, we will recall key features of mtFAO and its regulation.

mtFAO and Oxidative Phosphorylation

mtFAO and oxidative phosphorylation (OXPHOS) have been reviewed previously in details $[3,8,9]$. Briefly, mitochondria provide most of ATP by way of the oxidation of substrates such as FAs and pyruvate. Whereas pyruvate oxidation takes place in the tricarboxylic acid (TCA) cycle, mitochondrial degradation of FAs is mediated by the $\beta$-oxidation pathway. To this end, FAs must cross the mitochondrial membranes. Whereas short-chain and medium-chain fatty acids (SCFAs/MCFAs) freely enter the mitochondria and are then activated into acyl-CoA molecules by specific acyl-CoA synthetases, long-chain fatty acids (LCFAs) must cross the mitochondrial membranes with a multi-enzymatic system requiring both coenzyme A (CoA) and L-carnitine as cofactors. In this system, carnitine palmitoyltransferase 1 (CPT1) and CPT2 are playing a major role (Fig. 1). Inside mitochondria, acyl-CoA derivatives are cut down sequentially by the $\beta$-oxidation process that 
generates acetyl-CoA moieties and shorter fatty acids that enter new $\beta$-oxidation cycles (Fig. 1). These acetyl-CoA moieties subsequently generate ketone bodies (KB), which are used for ATP synthesis in extra-hepatic tissues. The key role of mtFAO in energy homeostasis is illustrated by the occurrence of multiple organ failure and death when this metabolic pathway is severely affected $[3,4,10]$.

mtFAO produces not only acetyl-CoA molecules but also $\mathrm{NADH}$ and $\mathrm{FADH}_{2}$ that provide their electrons and protons to the mitochondrial respiratory chain (MRC) (Fig. 1). This transfer of electrons and protons allows the regeneration of $\mathrm{NAD}^{+}$and $\mathrm{FAD}$, and the synthesis of ATP from ADP (Fig. 1). The whole process coupling substrate oxidation to ATP synthesis is called OXPHOS. OXPHOS uncouplers are drugs (or chemicals) that can reduce the mitochondrial membrane potential $\left(\Delta \psi_{\mathrm{m}}\right)$ and abolish ATP synthesis without inhibiting substrate oxidation $[3,4,11$, $12 \bullet \bullet, 13]$.

A unique feature of mitochondria is that $13 \mathrm{MRC}$ polypeptides are encoded by mitochondrial DNA (mtDNA) (Fig. 1). There are several hundred copies of mtDNA in a single cell, and mtDNA replication is carried out by the DNA polymerase $\gamma[3,5,8]$. In liver, it is deemed that mtDNA copy number must fall below 20-40\% of basal level in order to induce MRC impairment [14, 15], which can secondarily lead to reactive oxygen species (ROS) overproduction [16, 17]. Conversely, ROS and other endogenous molecules such as reactive nitrogen species (RNS) can subsequently damage mtDNA, thus leading to mtDNA mutations and depletion $[5,15,18]$.

\section{Regulation of the mtFAO Pathway}

During fasting, the expression of many enzymes involved in mtFAO is up-regulated by peroxisome proliferatoractivated receptor $\alpha(\operatorname{PPAR} \alpha)$, a transcription factor which can be activated by endogenous FAs [5, 9]. In addition, other transcription factors positively regulating hepatic FAO during fasting include forkhead box A2 (FoxA2) and cAMP-response element-binding protein (CREB) $[5,19]$. Moreover, the PPAR $\gamma$ coactivators $-1 \alpha$ and $1 \beta$ (PGC- $1 \alpha / \beta)$ are playing a key role in the transcriptional regulation of mtFAO enzymes [5, 9, 19]. After a meal, mtFAO of LCFAs can be inhibited by malonyl-CoA, since this intermediate of lipogenesis strongly inhibits CPT1 [3, 9].

In pathophysiological conditions, other negative regulations can exist. For instance, any significant reduction in CoA and L-carnitine levels can compromise mtFAO [3, 4, 20]. A strong reduction of MRC activity can also impair mtFAO. Indeed, inhibition of MRC activity slows down the oxidation of NADH and $\mathrm{FADH}_{2}$ into $\mathrm{NAD}^{+}$and FAD, which are mandatory cofactors for several mtFAO dehydrogenases [3, 21, 22]. Furthermore, any significant reduction of MRC activity can also impair the TCA cycle and cause lactic acidosis [4, 23].

Drug-Induced Inhibition of mtFAO

The main mechanisms whereby drugs are able to inhibit mtFAO can be classified into five different categories. It is noteworthy that different mechanisms can be involved for the same drug.

\section{Direct Inhibition of Mitochondrial $\beta$-Oxidation Enzyme(s)}

Some drugs (or their metabolites) can directly inhibit one or several enzyme(s) involved in mtFAO (Fig. 1). This has been showed with amiodarone, tamoxifen, perhexiline and valproic acid (VPA), or suspected with ibuprofen, amineptine and tianeptine [3-5, 24-29•]. For the latter three drugs, a stronger inhibition of mitochondrial $\beta$-oxidation of SCFAs and MCFAs compared to LCFAs suggested a specific impairment of enzymes involved in SCFA and MCFA oxidation, although the investigations did not determine the exact targeted enzyme(s) [24, 25].

Regarding VPA (dipropylacetic acid), severe inhibition of mtFAO is probably due to the generation of $\triangle 2,4-\mathrm{VPA}-$ $\mathrm{CoA}$ and other reactive metabolites that irreversibly inactivate FAO enzyme(s) [3, 30]. Interestingly, acetaminophen (APAP) could inhibit mtFAO and MRC activity via the generation of $N$-acetyl- $p$-benzoquinone imine (NAPQI) [31, 32], a reactive metabolite generated by cytochromes P450 3A4 and 2E1 (CYP2E1) [33]. This may explain, with other mechanisms (see below), why APAP intoxication can induce steatosis in rodents [34-36] and in some individuals (Table 1) [1••, 2].

Some investigations allowed the identification of the mtFAO enzyme(s) inhibited by the aforementioned drugs. For instance, it has been shown that CPT1 can be inhibited by VPA, amiodarone and tamoxifen [28, 29•, 37]. Troglitazone is able to inhibit long-chain acyl-CoA synthase (ACS) (Fig. 1), thus impairing the mitochondrial entry of LCFAs through a CPT1-independent mechanism [38].

\section{Sequestration of CoA and/or L-Carnitine}

Drugs such as VPA, salicylic acid and ibuprofen can impair mtFAO via the generation of CoA and/or L-carnitine esters, which decreases the availability of these cofactors for the $\beta$ oxidation of endogenous FAs (Fig. 1) [3, 5, 25, 39]. However, drug-induced inhibition of mtFAO secondary to CoA and/or L-carnitine depletion could occur only when cellular levels of these cofactors are already below physiological concentrations [25, 39]. For some drugs, generation of xenobiotic acyl-CoA thioesters could also competitively inhibit different mitochondrial enzyme(s) [40]. 


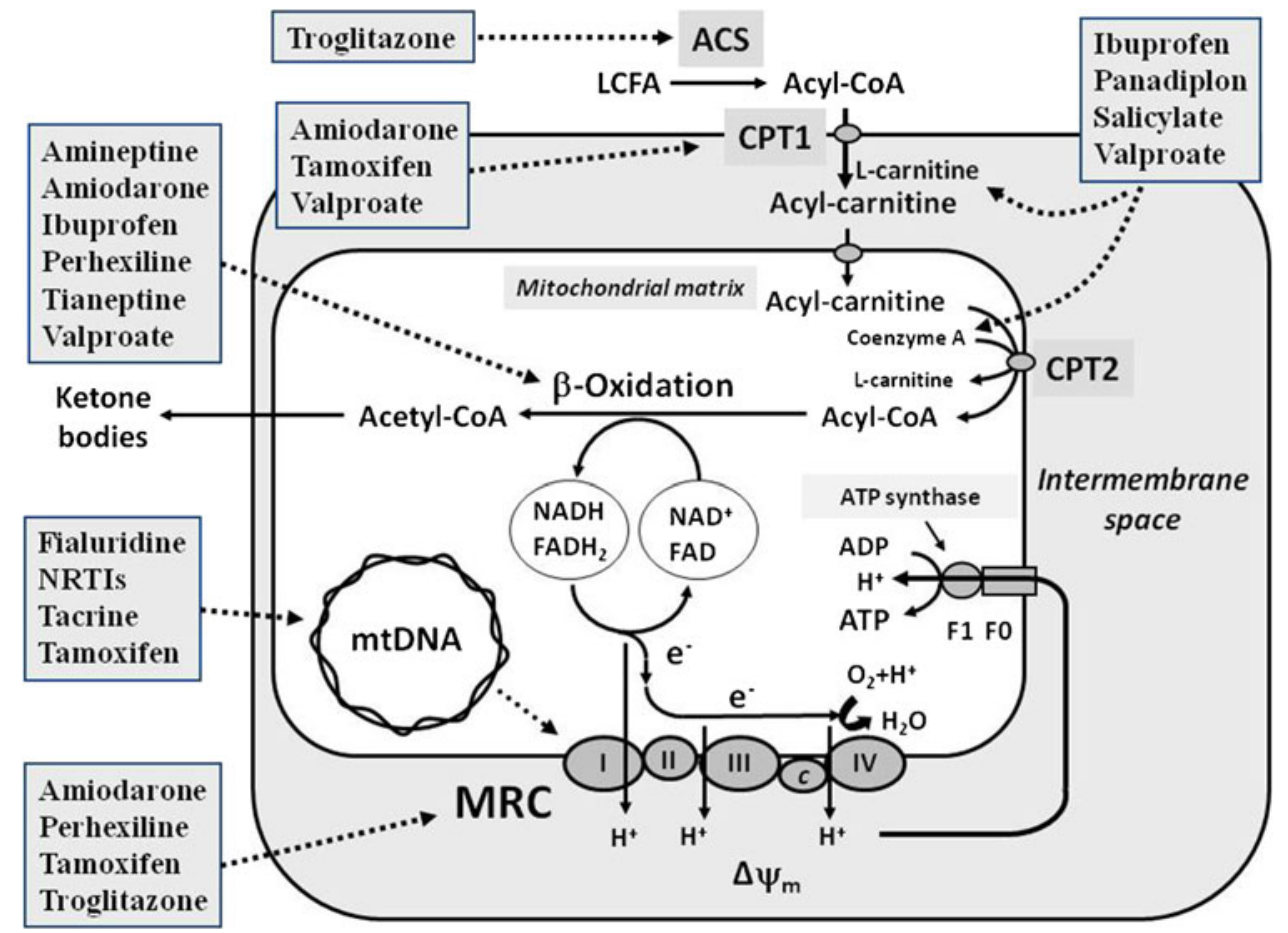

Fig. 1 Mitochondrial $\beta$-oxidation and main mechanisms whereby drugs can impair this metabolic pathway. Adapted from Begriche et al. [5]. Whereas short and medium-chain fatty acids (SCFAs and MCFAs) freely enter mitochondria (not shown), the entry of LCFAs within these organelles requires a specific shuttle system involving four steps. (1) LCFAs are activated into LCFA- CoA thioesters by long-chain acyl-CoA synthetases (ACS). (2) The LCFA-CoA is converted into an acyl-carnitine by carnitine palmitoyltransferase- 1 (CPT1) located in the outer mitochondrial membrane. (3) The acylcarnitine is transferred across the inner mitochondrial membrane into the mitochondrial matrix by carnitine-acylcarnitine translocase. (4) Finally, carnitine palmitoyltransferase-2 (CPT2), located on the inner side of the inner mitochondrial membrane, transfers the acyl moiety from carnitine back to CoA. Acyl-CoA thioesters are then oxidized into acetyl-CoA moieties via the $\beta$-oxidation process, irrespective of their chain length. Acetyl-CoA moieties can then generate KB (mainly acetoacetate and $\beta$-hydroxybutyrate), which are liberated into the plasma and used by extra-hepatic tissues for energy production. mtFAO generates NADH and $\mathrm{FADH}_{2}$, which transfer their electrons

\section{Inhibition of the MRC}

mtFAO can also be secondarily impaired as a result of severe inhibition of the MRC [3-5]. This could occur with amiodarone, perhexiline, tamoxifen and buprenorphine [4, $21,26,28,41]$. Interestingly, these amphiphilic drugs can be protonated within the mitochondrial intermembrane space, thus generating cationic compounds entering the matrix thanks to $\Delta \psi_{\mathrm{m}}$ (Fig. 1). This allows their accumulation within mitochondria and the subsequent inhibition of mtFAO and MRC enzymes. Actually, whereas relatively low concentrations of these amphiphilic drugs can inhibit directly FAO enzyme(s), higher concentrations are required to impair the respiratory chain $[11,21,26,28,41]$. $\left(\mathrm{e}^{-}\right)$to the mitochondrial respiratory chain (MRC), thus regenerating $\mathrm{NAD}^{+}$and FAD used for other $\beta$-oxidation cycles. Within the MRC, electrons are sequentially transferred to different polypeptide complexes (numbered from I to IV) embedded within the inner membrane. The final transfer of the electrons to oxygen takes place at the level of complex IV (cytochrome $c$ oxidase). Importantly, the flow of electrons within the MRC is coupled to the extrusion of protons $\left(\mathrm{H}^{+}\right)$from the matrix to the intermembrane space, which creates the mitochondrial transmembrane potential, $\Delta \psi_{\mathrm{m}}$. When energy is needed, these protons re-enter the matrix through ATP synthase (complex V), thus liberating energy that is used to phosphorylate ADP into ATP. The mitochondrial DNA (mtDNA) encodes 13 polypeptides, which are inserted within complexes I, III, IV and V. Drugs can impair mtFAO through different mechanisms such as: (1) direct inhibition of $\beta$-oxidation enzyme(s), including ACS, CPT1 and different acyl-CoA dehydrogenases; (2) sequestration of the mtFAO cofactors L-carnitine and CoA; (3) inhibition of MRC activity, either directly or indirectly by way of mtDNA depletion; (4) impairment of PPAR $\alpha$ expression and activity (not shown)

Thus, mitochondrial accumulation of these amphiphilic drugs eventually inhibits FAO through a dual mechanism. The precise sites of MRC inhibition have been identified for amiodarone and perhexiline, which both inhibit complexes I and II [21, 26].

For some drugs inhibiting mtFAO, investigations have not been able to clearly establish whether this deleterious effect was due to direct inhibition of mtFAO enzyme(s), or to indirect inhibition via MRC impairment. This is the case for tetracycline derivatives for which bone fine inhibition of mtFAO has been shown in some investigations [42, 43], while MRC (or OXPHOS) impairment has been demonstrated in other independent studies [3, 44, 45]. 
Table 1 Examples of drugs inducing microvesicular steatosis, macrovacuolar steatosis, steatohepatitis or cirrhosis in treated patients

Drugs in italics have been shown to impair mitochondrial $\beta$-oxidation and/or other key mitochondrial functions such as the MRC activity. Information concerning liver lesions can be found mainly in references [1-7] and [100]

$A C E$ angiotensin-converting enzyme, $C i r$ cirrhosis, $H B V$ hepatitis $\mathrm{B}$ virus, $H C V$ hepatitis $\mathrm{C}$ virus, $H I V$ human immunodeficiency virus, $\mathrm{MaSt}$ macrovacuolar steatosis, MiSt microvesicular steatosis, NSAID nonsteroidal anti-inflammatory drug, SERM selective estrogen receptor modulator, $\mathrm{StH}$ steatohepatitis

\begin{tabular}{|c|c|c|}
\hline Drug & Therapeutic class & Liver lesions \\
\hline Acetaminophen (overdose) & Analgesic, antipyretic & MiSt, MaSt \\
\hline Amineptine & Antidepressant & MiSt \\
\hline Amiodarone & Antianginal, antiarrhythmic & MiSt, MaSt, StH, Cir \\
\hline Aspirin (and salicylic acid) & NSAID & MiSt \\
\hline Carbamazepine & Antiepileptic & MaSt, StH, Cir \\
\hline Diclofenac & NSAID & MaS, Cir \\
\hline Didanosine (ddI) & Antiretroviral (anti-HIV) & MiSt, MaSt, StH, Cir \\
\hline Enalapril & Antihypertensive (ACE inhibitor) & MiSt \\
\hline Fialuridine (FIAU) & Antiviral (anti-HBV) & MiSt \\
\hline 5-Fluorouracil & Antineoplastic (colorectal cancer) & MaSt, Cir \\
\hline Glucocorticoids & Anti-inflammatory & MaSt \\
\hline Ibuprofen & NSAID & MiSt, MaSt, Cir \\
\hline Indinavir & Antiretroviral (anti-HIV) & MiSt \\
\hline Interferon- $\alpha$ & Antiviral (anti-HCV and anti-HBV) & MaSt \\
\hline Irinotecan & Antineoplastic (colorectal cancer) & MaSt, StH \\
\hline Methotrexate & Antipsoriatic, anti-rheumatoid & MaSt, StH, Cir \\
\hline Nifedipine & Antianginal, antihypertensive & MaSt, StH \\
\hline Panadiplon & Anxiolytic & MiSt \\
\hline Perhexiline & Antianginal & MaSt, StH, Cir \\
\hline Pirprofen & NSAID & MiSt \\
\hline Raloxifene & SERM, anti-osteoporotic & MaSt \\
\hline Stavudine $(d 4 T)$ & Antiretroviral (anti-HIV) & MiSt, MaSt, StH, Cir \\
\hline Tamoxifen & SERM, antineoplastic (breast cancer) & MaSt, StH, Cir \\
\hline $\begin{array}{l}\text { Tetracycline and its } \\
\text { derivatives (high doses) }\end{array}$ & Antibiotics & MiSt \\
\hline Tianeptine & Antidepressant & MiSt \\
\hline Toremifene & SERM, antineoplastic (breast cancer) & MaSt \\
\hline Troglitazone & Antidiabetic & MiSt \\
\hline Valproic acid & Antiepileptic & MiSt \\
\hline Zidovudine (AZT) & Antiretroviral (anti-HIV) & MiSt \\
\hline
\end{tabular}

Drugs such as tianeptine and ibuprofen inhibit MRC activity, in particular at the level of complex I [46-48]. Importantly, these effects were demonstrated on heart, duodenum or brain mitochondria. However, some investigations showed that mitochondrial toxicity could greatly vary between tissues [15, 49]. Actually, ibuprofen and tianeptine-induced impairment of complex $I$ in liver mitochondria is unlikely to be strong because this would cause a similar inhibition of $\beta$-oxidation with all kinds of FAs (i.e., SCFAs, MCFAs and LCFAs), thus irrespective of their chain length. However, investigations performed on isolated mouse liver mitochondria showed that these drugs inhibited more strongly the $\beta$-oxidation of SCFAs and MCFAs compared to LCFAs [24, 25]. Accordingly, although ibuprofen inhibited mitochondrial respiration with glutamate/malate on isolated liver mitochondria (Table 2) [12••], this effect appeared with much higher concentrations compared to those affecting MRC activity with mitochondria isolated from duodenum [48].

\section{Impairment of mtDNA Replication}

Inhibition of mtFAO can also result from reduced hepatic mtDNA levels. This mechanism has been shown for the antiviral fialuridine (FIAU), zidovudine (AZT), stavudine (d4T) and didanosine (ddI), which all inhibit mtDNA polymerase $\gamma$ activity [3-5, 50, 51]. Importantly, liver injury induced by these drugs can be associated with severe lactic acidosis, which is caused by TCA cycle inhibition. Interestingly, tamoxifen and tacrine can induce hepatic mtDNA depletion possibly by interacting with the mitochondrial topoisomerases [28, 52].

Finally, some drugs could also induce mtDNA depletion via the generation of ROS, RNS and/or reactive metabolites. For instance, APAP and troglitazone can reduce mtDNA levels by inducing mtDNA strand breaks [53, 54]. Indeed, damaged mtDNA molecules harboring numerous strand breaks (or other bulky damages) can be rapidly degraded by mitochondrial endonucleases $[18,55]$. 
Table 2 Drug-induced inhibition of mitochondrial respiration with glutamate/malate and palmitoyl-L-carnitine/malate in liver mitochondria isolated from lean and ob/ob mice

\begin{tabular}{|c|c|c|c|c|}
\hline \multirow[t]{2}{*}{ Drugs } & \multicolumn{2}{|l|}{ Lean mice } & \multicolumn{2}{|l|}{ Ob/ob mice } \\
\hline & Glutamate + malate & Palmitoyl-L-carnitine + malate & Glutamate + malate & Palmitoyl-L-carnitine + malate \\
\hline Acetaminophen (APAP) & $>400^{\mathrm{a}}$ & $>400$ & $>400$ & $>400$ \\
\hline Carbamazepine & $>400$ & $>400$ & 379 & 341 \\
\hline Diclofenac & 35 & 47 & $16^{*}$ & $11 *$ \\
\hline Ibuprofen & 107 & 287 & 99 & $80 *$ \\
\hline Irinotecan & 10 & 6 & 25 & 10 \\
\hline Methotrexate & 53 & 44 & 40 & 42 \\
\hline Salicylic acid & $>400$ & $>400$ & $>400$ & $>400$ \\
\hline Tamoxifen & 42 & 4 & 72 & 11 \\
\hline Zidovudine (AZT) & 309 & 79 & 144 & 83 \\
\hline
\end{tabular}

Measurement of oxygen consumption in the presence of ADP (state 3) and the different substrates was carried out on the Mitologics screening

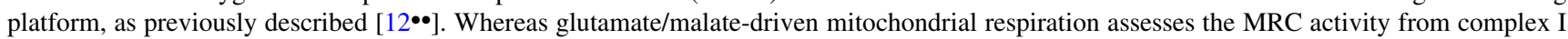
to complex IV, palmitoyl-L-carnitine/malate-driven respiration evaluates LCFA mtFAO

* Different from lean mice $(P<0.05)$

${ }^{a}$ Numbers in this table correspond to the effective concentrations (in $\left.\mu \mathrm{M}\right)$ inducing $20 \%$ of the maximal effects $\left(\mathrm{EC}_{20}\right)$ as described in $[12 \bullet \bullet]$. Values are means for 3-5 different mitochondrial preparations

\section{Impaired PPAR $\alpha$ Activity}

Some drugs could impair mtFAO by reducing PPAR $\alpha$ expression and activity. This is suspected with APAP, VPA and tetracycline that reduce the mRNA expression of PPAR $\alpha$ and some of its target genes including CPT1 [36, $44,56]$. Although the direct consequences of this effect are still uncertain, impairing PPAR $\alpha$ activity could prevent an important metabolic adaptation that takes place during drug-induced steatosis [57, 58]. The mechanism(s) whereby these drugs could impair PPAR $\alpha$ expression might deserve further attention.

Drug-Induced Alterations of Other Pathways Involved in Lipid Homeostasis

Although not being in the scope of this article, it is noteworthy that drug-induced steatosis can be also caused by other mechanisms. For instance, drugs can inhibit very low density lipoprotein (VLDL) secretion and increase lipid synthesis, in particular by direct (or indirect) activation of key lipogenic transcription factors such as sterol regulatory element binding protein 1c (SREBP-1c) and PPAR $\gamma[5,59$, 60]. Interestingly, some drugs such as amiodarone and tamoxifen could both inhibit mtFAO and stimulate de novo lipogenesis [5, 61•].

\section{Drug-Induced Microvesicular Steatosis}

Numerous investigations have shown that drug-induced microvesicular steatosis is the consequence of severe inhibition of mtFAO [3-7, 21, 24, 25, 28, 29•, 39, 42, 62].
Interestingly, this mechanism is also involved in the pathophysiology of microvesicular steatosis occurring in other conditions such as inborn errors of mtFAO, Reye's syndrome and acute fatty liver of pregnancy $[3,10]$.

A primary consequence of severe inhibition of mtFAO is ATP depletion and accumulation of FAs that are either esterified into triglycerides, or that remain as a free form $[3$, 13]. It has been postulated that the small size of the lipid droplets could be due to an "emulsification" of triglycerides by free fatty acids [3], although this hypothesis has never been confirmed. Alternatively, the nature and/or the abundance of some proteins wrapping the lipids could play a role [5, 63]. Whereas triglycerides are not toxic for the cells, free FAs and some of their derivatives (i.e., acyl-CoA thioesters and dicarboxylic acids) could worsen mitochondrial dysfunction and cause cell death [3, 9, 64].

Drug-induced microvesicular steatosis is a potentially severe and fatal liver lesion that can be associated with liver failure, encephalopathy and profound hypoglycemia [3-5]. Liver pathology shows the presence within the cytoplasm of numerous lipid droplets, which leave the nucleus in the center of the hepatocyte [3, 21, 42, 62]. Besides lipid accumulation, hepatic cytolysis and increased plasma transaminases can also be observed to a variable degree. Examples of drugs able to induce microvesicular steatosis are given in Table 1 [3-5, 65-68].

Drug-induced microvesicular steatosis can be associated with severe hypoglycemia and abnormal levels of plasma KB [3-5]. Hypoglycemia could be due to impaired gluconeogenesis and/or to higher extra-hepatic utilization of glucose [3, 69]. Although hypoketonemia has been observed with VPA, pirprofen and ibuprofen, high levels of plasma 
KB was also reported experimentally with amineptine, amiodarone, salicylic acid, tetracycline and tianeptine [3, 4, 21, 24, 39, 42, 70]. Drug-induced hyperketonemia could be related to a severe inhibition of peripheral $\mathrm{KB}$ utilization $[3,4]$. Finally, microvesicular steatosis can be associated with an accumulation of acyl-carnitine derivatives and dicarboxylic acids in plasma and urine [3-5].

Inhibition of mtFAO and Steatosis Induced by Other Xenobiotics

Although beyond the scope of this review, it is noteworthy that nonpharmaceutical compounds are able to inhibit mtFAO and induce hepatic lipid accumulation, especially as microvesicular steatosis. This is for instance the case with alcohol, cocaine, perfluorooctane sulfonate (a persistent organic pollutant), triptolide (a diterpenoid epoxide isolated from a Chinese woody wine plant), and hypoglycine (a toxin present in the unripe fruit of Jamaican ackee tree) $[3,55,71 \cdot, 72,73]$.

Factors Favoring Drug-Induced Mitochondrial Dysfunction

At least three factors could favor drug-induced impairment of mtFAO and MRC activity. Importantly, these factors are not mutually exclusive and their combination is likely to induce severe mitochondrial dysfunction and microvesicular steatosis in some patients.

\section{Drug Structure and Biotransformation}

Amiodarone, perhexiline and tamoxifen are amphiphilic drugs harboring protonable amine moieties that favor their accumulation inside the mitochondrial matrix $[3,7,11,26$, 28]. For amiodarone, the benzofuranyl-phenylmethanone moiety could be the chemical structure responsible for mitochondrial dysfunction [74-76•]. VPA is a branchedchain fatty acid that freely enters the mitochondria, where it is activated by $\mathrm{CoA}$ and undergoes $\beta$-oxidation $[3,6,40]$. However, the two-step biotransformation of VPA by CYPs and $\beta$-oxidation generates reactive metabolites that irreversibly inactivate FAO enzymes and induce cytotoxicity $[3,30,77]$. Regarding the role of CYPs, it is noteworthy that APAP-induced dysfunction of liver mitochondria could depend, at least in part, on the presence of CYP2E1 within these organelles [33]. Finally, the antiretroviral nucleoside reverse transcriptase inhibitors (NRTIs) are able to inhibit mtDNA replication and cause severe mtDNA depletion owing to their structural analogy with the natural nucleosides $[3,6,15]$. In this pharmacological class, drugs such as zalcitabine (ddC), ddI and $\mathrm{d} 4 \mathrm{~T}$ are significantly more toxic to mitochondria than others (AZT and lamivudine) [78].

\section{Drug Dosage and Duration of Treatment}

Clinical reports in the 50s and 60s indicated that severe microvesicular steatosis induced by tetracycline and its derivatives was clearly dose-dependent [3]. In particular, most cases of steatosis were observed in patients receiving large intravenous dosages $(>1.5 \mathrm{~g} /$ day) of tetracycline derivatives [3]. However, tetracycline-induced steatosis is no longer observed since such huge intravenous doses have been abandoned. Long-lasting administration of NRTIs also increases the risk of mitochondrial toxicity in liver and other tissues [15, 79].

\section{Genetic Predispositions}

Several congenital defects in mtFAO and OXPHOS enzymes have been detected in patients with VPA-induced hepatotoxicity $[3,5,80]$. A mutation in the gene encoding DNA polymerase $\gamma$ (POLG) could favor mitochondrial toxicity induced by NRTIs, possibly by enhancing the probability of their incorporation within the mtDNA molecules and the subsequent arrest of mtDNA replication [5, 81]. Intriguingly, mutations in the POLG gene could also favor VPA-induced hepatotoxicity [82•], although this cannot be explained by the incorporation of VPA within mtDNA. Finally, interindividual differences in mitochondrial antioxidant enzymes such as MnSOD could enhance the risk of mitochondrial toxicity and liver injury [83, 84].

\section{Drug-Induced Macrovacuolar Steatosis} and Steatohepatitis

With some drugs, liver triglycerides accumulate as a large (often single) lipid vacuole displacing the nucleus close to the plasma membrane. This lesion is commonly referred to as macrovacuolar steatosis and can be induced by other factors such as high-calorie feeding and ethanol intoxication [4, 83, 85]. Examples of drugs able to induce macrovacuolar steatosis are indicated in Table 1 [4, 5, 86-88].

The prevalence of drug-induced macrovacuolar steatosis may be underestimated, mainly because this liver lesion is benign, at least in the short term. However, this lesion can progress in the long term to steatohepatitis, which is characterized by necrosis, inflammation and some fibrosis. Moreover, some drugs such as amiodarone, perhexiline, tamoxifen and ddI can also induce cirrhosis after long-term

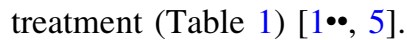

In contrast to microvesicular steatosis, whicht can be considered as a bona fide mitochondrial disease, several mechanisms seem to be involved in the pathogenesis of drug-induced macrovacuolar steatosis. These mechanisms include moderate inhibition of mtFAO, enhanced de novo lipogenesis and reduced secretion of VLDL [5, 59, 60]. 
Importantly, these different mechanisms are not mutually exclusive, and for instance some drugs can inhibit both $\mathrm{mtFAO}$ and VLDL secretion [5, 60].

It is also noteworthy that some drugs can induce both microvesicular and macrovacuolar steatosis (Table 1). Although the exact reason of this observation is unclear, it is conceivable that microvesicular steatosis could occur when mtFAO is severely inhibited, whereas macrovacuolar steatosis could take place if mitochondrial function is relatively preserved [5]. The differences in the severity of drug-induced mitochondrial dysfunction can be explained by several factors, as discussed in the previous section.

Although the pathophysiology of drug-induced steatohepatitis is not fully understood, some data suggest that reduced MRC activity could be involved [4, 5, 26, 89]. Indeed, MRC inhibition could not only contribute to fat deposition but also to ATP deficiency, which can cause necrosis. Moreover, MRC impairment can be associated with ROS overproduction, a key event involved in the progression of fatty liver to steatohepatitis, whatever its etiology [5, 9, 89]. Importantly, ROS overproduction in a cellular environment enriched in fat can trigger lipid peroxidation and the production of reactive aldehydes that induce many deleterious effects in liver [5, 9, 89, 90]. Although drug-induced inhibition of MRC and $\beta$-oxidation mostly leads to the hepatic accretion of saturated fatty acids, some polyunsaturated fatty acids can also accumulate in sufficient quantity to generate lipid peroxidationderived reactive aldehydes in the presence of ROS [71].

\section{Drug-Induced Inhibition of mtFAO and Aggravation of NAFLD}

Obesity is often associated with metabolic disorders such as nonalcoholic fatty liver disease (NAFLD), dyslipidemia, and type 2 diabetes [5, 9]. During NAFLD, insulin resistance and adaptive hyperinsulinemia favor fat deposition in the liver, in particular via SREBP1c-mediated de novo lipogenesis [9]. However, there is a compensatory stimulation of mtFAO in order to limit fat accretion $[9,85,91]$. Thus, any significant impairment of mtFAO is likely to aggravate NAFLD in obese individuals. Moreover, drugs that alter MRC activity are also likely to promote the progression of fatty liver to nonalcoholic steatohepatitis (NASH) by enhancing ROS generation and oxidative stress [59].

Drugs that are suspected to aggravate NAFLD in obese patients are tamoxifen, raloxifene, irinotecan, methotrexate and NRTIs such as d4T and ddI [9, 59, 92]. Interestingly, inhibition of mtFAO has been documented with several of these drugs, namely tamoxifen, raloxifene and NRTIs [3, 28, 93, 94]. Moreover, drugs such as tamoxifen, methotrexate and NRTIs can inhibit MRC activity and favor oxidative stress $[3,16,28,95,96]$. However, for some of these compounds, aggravation of preexisting
NAFLD could also be secondary to other mechanisms. For instance, tamoxifen could also inhibit VLDL secretion and stimulate de novo lipogenesis [28, 59, 97].

We took advantage of the present article to determine whether $\beta$-oxidation of palmitoyl-L-carnitine could be more severely inhibited by some of the afore-mentioned drugs when liver mitochondria were isolated from ob/ob mice compared to wild-type mice. Although irinotecan, methotrexate and tamoxifen impaired palmitoyl-L-carnitine $\beta$-oxidation, this inhibition was not statistically different between wild-type and ob/ob liver mitochondria (Table 2). Nevertheless, further investigations will be required to determine whether chronic administration of these drugs could aggravate fatty liver in ob/ ob mice. In contrast, other drugs were able to inhibit palmitoyl-L-carnitine $\beta$-oxidation more strongly on ob/ob liver mitochondria, as discussed below.

\section{Conclusion and Outlook}

As discussed in this review, inhibition of mtFAO is a key mechanism whereby drugs can induce steatosis, and actually drug-induced microvesicular steatosis can be considered as a mitochondrial disease [3-7]. Moreover, long-term impairment of MRC activity could be an important mechanism leading to drug-induced steatohepatitis, in particular as a result of mitochondrial ROS overproduction [5, 26, 89]. It is also noteworthy that some drugs able to induce mitochondrial dysfunction could be more toxic in obese patients with preexisting NAFLD, which could be aggravated during the treatment. Indeed, drug-induced mtFAO impairment can impede a key compensatory metabolic pathway set up during NAFLD in order to limit hepatic fat accumulation [9, 59, 91], whereas MRC impairment is able to major oxidative stress and lipid peroxidation [26, 89].

Although numerous drugs can induce steatosis [1, 3, 4], their ability to inhibit mtFAO is still unknown for a majority of them. Thus, high-throughput screening can be suited in order to determine whether inhibition of mtFAO is a frequent feature observed with all the steatogenic drugs. Importantly, such screening could also assess the ability of these drugs to impair MRC activity [12••, 98•]. These investigations can also be performed on liver mitochondria isolated from obese and wild-type mice in order to determine whether "obese" mitochondria are more sensitive to drug-induced mitochondrial dysfunction. Table 2 gives some examples of steatogenic drugs and their ability to inhibit (or not) mitochondrial respiration assessed with glutamate/malate and palmitoyl-L-carnitine/malate on liver mitochondria isolated from lean and ob/ob mice.

These investigations provided some new interesting findings. For instance, diclofenac and ibuprofen-induced inhibition of mtFAO was stronger with ob/ob mitochondria (Table 2). Importantly, this stronger impairment was not due 
to lower basal oxygen consumption with palmitoyl-L-carnitine since it was increased by $62 \%$ in ob/ob mitochondria as compared to wild-type mitochondria, in keeping with previous investigations [9, 91]. Moreover, diclofenac induced a stronger inhibition of glutamate/malate-driven respiration on ob/ob mitochondria. It would be interesting to determine whether these two NSAIDs could worsen fatty liver in ob/ob mice, or in other murine models of obesity and NAFLD. Our investigations also revealed that irinotecan strongly inhibited mtFAO and MRC activity (Table 2). Although more investigations will be needed, these novel data could explain why this antineoplastic drug is able to induce steatohepatitis in some patients (Table 1) [2, 99]. Finally, we found that AZT inhibited palmitoyl-L-carnitine-driven respiration at concentrations below those required to impair MRC (Table 1). Interestingly, some investigations already showed that this antiretroviral drug was able to directly impair MRC activity (e.g. complex II), in addition to its long-term deleterious effect on mtDNA replication [15]. Thus, AZT could also directly inhibit mtFAO in liver, in addition to its detrimental effects on MRC activity.

Acknowledgments This work was supported by INSERM (Institut Nationale de la Santé et de la Recherche Médicale) and ANR (Agence Nationale de la Recherche; PHARMECO Project from the CES program). Julie Massart was supported by a scholarship from the Swedish Institute.

Compliance with Ethics Guidelines This article does not contain any studies with human or animal subjects performed by any of the authors.

Disclosure Julie Massart and Karima Begriche declare that they have no conflict of interest. Nelly Buron, Mathieu Porceddu and Annie Borgne-Sanchez are co-founders of Mitologics SAS. Bernard Fromenty declares that he has no conflict of interest. He has received consulting fees from Medicines for Malaria Venture and Medicen Paris Region, and research funding from Société Francophone du Diabète.

\section{References}

Papers of particular interest have been highlighted as:

- Of importance

•- Of major importance

1. • Biour M, Ben Salem C, Chazouillères O, et al (2004) Druginduced liver injury; fourteenth updated edition of the bibliographic database of liver injuries and related drugs. Gastroenterol Clin Biol 28:720-759. This article provides an essential database concerning DILI for more than a thousand pharmaceuticals. Importantly, this French database is now available on line (http://server1.dicofm. com/hepatox/index.php) and is regularly updated. Another important database regarding DILI is the FDA's Liver Toxicity Knowledge Base (LTKB) (http://www.fda.gov/ScienceResearch/ BioinformaticsTools/LiverToxicityKnowledgeBase).

2. Ramachandran R, Kakar S (2009) Histological patterns in druginduced liver disease. J Clin Pathol 62:481-492
3. Fromenty B, Pessayre D (1995) Inhibition of mitochondrial beta-oxidation as a mechanism of hepatotoxicity. Pharmacol Ther 67:101-154

4. Labbe G, Pessayre D, Fromenty B (2008) Drug-induced liver injury through mitochondrial dysfunction: mechanisms and detection during preclinical safety studies. Fundam Clin Pharmacol 22:335-353

5. Begriche K, Massart J, Robin MA et al (2011) Drug-induced toxicity on mitochondria and lipid metabolism: mechanistic diversity and deleterious consequences for the liver. J Hepatol 54:773-794

6. Pessayre D, Mansouri A, Berson A et al (2010) Mitochondrial involvement in drug-induced liver injury. Handb Exp Pharmacol 196:311-365

7. Pessayre D, Fromenty B, Berson A et al (2012) Central role of mitochondria in drug-induced liver injury. Drug Metab Rev 44:34-87

8. Wallace DC, Fan W, Procaccio V (2010) Mitochondrial energetics and therapeutics. Annu Rev Pathol 5:297-348

9. Begriche K, Massart J, Robin MA et al (2013) Mitochondrial adaptations and dysfunctions in nonalcoholic fatty liver disease. Hepatology (in press)

10. Shekhawat PS, Matern D, Strauss AW (2005) Fetal fatty acid oxidation disorders, their effect on maternal health and neonatal outcome: impact of expanded newborn screening on their diagnosis and management. Pediatr Res 57:78R-86R

11. Fromenty B, Fisch C, Berson A et al (1990) Dual effect of amiodarone on mitochondrial respiration. Initial protonophoric uncoupling effect followed by inhibition of the respiratory chain at the levels of complex I and II. J Pharmacol Exp Ther 255:1377-1384

12. •• Porceddu M, Buron N, Roussel C, et al (2012) Prediction of liver injury induced by chemicals in human with a multiparametric assay on isolated mouse liver mitochondria. Toxicol Sci. 129:332-345. This article reports the effects of more than a hundred drugs on different mitochondrial parameters including oxygen consumption, membrane potential, swelling and cytochrome c release. The data revealed a highly significant relationship between mitochondrial toxicity and the occurrence of drug-induced liver injury in human.

13. Fromenty B, Letteron P, Fisch C et al (1993) Evaluation of human blood lymphocytes as a model to study the effects of drugs on human mitochondria. Effects of low concentrations of amiodarone on fatty acid oxidation, ATP levels and cell survival. Biochem Pharmacol 46:421-432

14. Ducluzeau PH, Lachaux A, Bouvier R et al (2002) Progressive reversion of clinical and molecular phenotype in a child with liver mitochondrial DNA depletion. J Hepatol 36:698-703

15. Igoudjil A, Begriche K, Pessayre D et al (2006) Mitochondrial, metabolic and genotoxic effects of antiretroviral nucleoside reverse-transcriptase inhibitors. Curr Med Chem Anti Infect Agents 5:273-292

16. Velsor LW, Kovacevic M, Goldstein M et al (2004) Mitochondrial oxidative stress in human hepatoma cells exposed to stavudine. Toxicol Appl Pharmacol 199:10-19

17. Golubitzky A, Dan P, Weissman S et al (2011) Screening for active small molecules in mitochondrial complex I deficient patient's fibroblasts, reveals AICAR as the most beneficial compound. PLoS ONE 6:e26883

18. Kazak L, Reyes A, Holt IJ (2012) Minimizing the damage: repair pathways keep mitochondrial DNA intact. Nat Rev Mol Cell Biol 13:659-671

19. Wolfrum C, Stoffel M (2006) Coactivation of Foxa2 through PGC-1 $\beta$ promotes liver fatty acid oxidation and triglyceride/ VLDL secretion. Cell Metab 3:99-110

20. Knapp AC, Todesco L, Torok M et al (2008) Effect of carnitine deprivation on carnitine homeostasis and energy metabolism in 
mice with systemic carnitine deficiency. Ann Nutr Metab 52:136-144

21. Fromenty B, Fisch C, Labbe G et al (1990) Amiodarone inhibits the mitochondrial $\beta$-oxidation of fatty acids and produces microvesicular steatosis of the liver in mice. J Pharmacol Exp Ther 255:1371-1376

22. Berson A, Schmets L, Fisch C et al (1994) Inhibition by nilutamide of the mitochondrial respiratory chain and ATP formation. Possible contribution to the adverse effects of this antiandrogen. J Pharmacol Exp Ther 270:167-176

23. Dykens JA, Jamieson J, Marroquin L et al (2008) Biguanideinduced mitochondrial dysfunction yields increased lactate production and cytotoxicity of aerobically-poised HepG2 cells and human hepatocytes in vitro. Toxicol Appl Pharmacol 233:203-210

24. Fromenty B, Fréneaux E, Labbe G et al (1989) Tianeptine, a new tricyclic antidepressant metabolized by beta-oxidation of its heptanoic side chain, inhibits the mitochondrial oxidation of medium and short chain fatty acids in mice. Biochem Pharmacol 38:3743-3751

25. Fréneaux E, Fromenty B, Berson A et al (1990) Stereoselective and nonstereoselective effects of ibuprofen enantiomers on mitochondrial beta-oxidation of fatty acids. J Pharmacol Exp Ther 255:529-535

26. Deschamps D, DeBeco V, Fisch C et al (1994) Inhibition by perhexiline of oxidative phosphorylation and the $\beta$-oxidation of fatty acids: possible role in pseudoalcoholic liver lesions. Hepatology 19:948-961

27. Baldwin GS, Murphy VJ, Yang Z et al (1998) Binding of nonsteroidal antiinflammatory drugs to the $\alpha$-subunit of the trifunctional protein of long chain fatty acid oxidation. J Pharmacol Exp Ther 286:1110-1114

28. Larosche I, Lettéron P, Fromenty B et al (2007) Tamoxifen inhibits topoisomerases, depletes mitochondrial DNA, and triggers steatosis in mouse liver. J Pharmacol Exp Ther 321:526-535

29. - Aires CC, Ijlst L, Stet F, Prip-Buus C, et al (2010) Inhibition of hepatic carnitine palmitoyl-transferase I (CPT IA) by valproyl$\mathrm{CoA}$ as a possible mechanism of valproate-induced steatosis. Biochem Pharmacol. 79:792-799. This article showed that valproyl-CoA is inhibiting CPT1, a key enzyme involved in the mitochondrial $\beta$-oxidation of LCFAs. Many other investigations from this group deciphered the mitochondrial toxicity of this antiepileptic drug (see for instance references 30 and 40).

30. Silva MF, Ruiter JP, Ijlst L et al (2001) Differential effect of valproate and its Delta2- and Delta4-unsaturated metabolites, on the beta-oxidation rate of long-chain and medium-chain fatty acids. Chem Biol Interact 137:203-212

31. Chen C, Krausz KW, Shah YM et al (2009) Serum metabolomics reveals irreversible inhibition of fatty acid $\beta$-oxidation through the suppression of PPAR $\alpha$ activation as a contributing mechanism of acetaminophen-induced hepatotoxicity. Chem Res Toxicol 22:699-707

32. Burcham PC, Harman AW (1991) Acetaminophen toxicity results in site-specific mitochondrial damage in isolated mouse hepatocytes. J Biol Chem 266:5049-5054

33. Knockaert L, Descatoire V, Vadrot N et al (2011) Mitochondrial CYP2E1 is sufficient to mediate oxidative stress and cytotoxicity induced by ethanol and acetaminophen. Toxicol In Vitro $25: 475-484$

34. Huang Q, Jin X, Gaillard ET et al (2004) Gene expression profiling reveals multiple toxicity endpoints induced by hepatotoxicants. Mutat Res 549:147-167

35. Yohe HC, O'Hara KA, Hunt JA et al (2006) Involvement of Toll-like receptor 4 in acetaminophen hepatotoxicity. Am J Physiol Gastrointest Liver Physiol 290:G1269-G1279
36. Aubert J, Begriche K, Delannoy M et al (2012) Differences in early acetaminophen hepatotoxicity between obese ob/ob and $\mathrm{db} / \mathrm{db}$ mice. J Pharmacol Exp Ther 342:676-687

37. Kennedy JA, Unger SA, Horowitz JD (1996) Inhibition of carnitine palmitoyltransferase- 1 in rat heart and liver by perhexiline and amiodarone. Biochem Pharmacol 52:273-280

38. Fulgencio JP, Kohl C, Girard J et al (1996) Troglitazone inhibits fatty acid oxidation and esterification, and gluconeogenesis in isolated hepatocytes from starved rats. Diabetes 45:1556-1562

39. Deschamps D, Fisch C, Fromenty B et al (1991) Inhibition by salicylic acid of the activation and thus oxidation of long chain fatty acids. Possible role in the development of Reye's syndrome. J Pharmacol Exp Ther 259:894-904

40. Luís PB, Ruiter JP, Ofman R et al (2011) Valproic acid utilizes the isoleucine breakdown pathway for its complete $\beta$-oxidation. Biochem Pharmacol 82:1740-1746

41. Berson A, Fau D, Fornacciari R et al (2001) Mechanisms for experimental buprenorphine hepatotoxicity: major role of mitochondrial dysfunction versus metabolic activation. J Hepatol 34:261-269

42. Fréneaux E, Labbe G, Letteron P et al (1988) Inhibition of the mitochondrial oxidation of fatty acids by tetracycline in mice and in man: possible role in microvesicular steatosis induced by this antibiotic. Hepatology 8:1056-1062

43. Labbe G, Fromenty B, Freneaux E et al (1991) Effects of various tetracycline derivatives on in vitro and in vivo beta-oxidation of fatty acids, egress of triglycerides from the liver, accumulation of hepatic triglycerides, and mortality in mice. Biochem Pharmacol 41:638-641

44. Yu HY, Wang BL, Zhao J et al (2009) Protective effect of bicyclol on tetracycline-induced fatty liver in mice. Toxicology 261:112-118

45. Cuenca-Lopez MD, Karachitos A, Massarotto L et al (2012) Minocycline exerts uncoupling and inhibiting effects on mitochondrial respiration through adenine nucleotide translocase inhibition. Pharmacol Res 65:120-128

46. Abdel-Razaq W, Kendall DA, Bates TE (2011) The effects of antidepressants on mitochondrial function in a model cell system and isolated mitochondria. Neurochem Res 36:327-338

47. Hroudová J, Fišar Z (2012) In vitro inhibition of mitochondrial respiratory rate by antidepressants. Toxicol Lett 213:345-352

48. Sandoval-Acuña C, Lopez-Alarcón C, Aliaga ME et al (2012) Inhibition of mitochondrial complex I by various non-steroidal anti-inflammatory drugs and its protection by quercetin via a coenzyme Q-like action. Chem Biol Interact 199:18-28

49. Gunter TE, Gerstner B, Lester T et al (2010) An analysis of the effects of $\mathrm{Mn}^{2+}$ on oxidative phosphorylation in liver, brain, and heart mitochondria using state 3 oxidation rate assays. Toxicol Appl Pharmacol 249:65-75

50. Lewis W, Levine ES, Griniuviene B et al (1996) Fialuridine and its metabolites inhibit DNA polymerase $\gamma$ at sites of multiple adjacent analog incorporation, decrease mtDNA abundance, and cause mitochondrial structural defects in cultured hepatoblasts. Proc Natl Acad Sci USA 93:3592-3597

51. Walker UA, Venhoff $\mathrm{N}$ (2005) Uridine in the prevention and treatment of NRTI-related mitochondrial toxicity. Antivir Ther 10(Suppl 2):M117-M123

52. Mansouri A, Haouzi D, Descatoire V et al (2003) Tacrine inhibits topoisomerases and DNA synthesis to cause mitochondrial DNA depletion and apoptosis in mouse liver. Hepatology 38:715-725

53. Cover C, Mansouri A, Knight TR et al (2005) Peroxynitriteinduced mitochondrial and endonuclease-mediated nuclear DNA damage in acetaminophen hepatotoxicity. J Pharmacol Exp Ther 315:879-887

54. Rachek LI, Yuzefovych LV, Ledoux SP et al (2009) Troglitazone, but not rosiglitazone, damages mitochondrial DNA and 
induces mitochondrial dysfunction and cell death in human hepatocytes. Toxicol Appl Pharmacol 240:348-354

55. Demeilliers C, Maisonneuve C, Grodet A et al (2002) Impaired adaptive resynthesis and prolonged depletion of hepatic mitochondrial DNA after repeated alcohol binges in mice. Gastroenterology 123:1278-1290

56. Wang W, Lin R, Zhang J et al (2012) Involvement of fatty acid metabolism in the hepatotoxicity induced by divalproex sodium. Hum Exp Toxicol 31:1092-1101

57. McCarthy TC, Pollak PT, Hanniman EA et al (2004) Disruption of hepatic lipid homeostasis in mice after amiodarone treatment is associated with peroxisome proliferator-activated receptor- $\alpha$ target gene activation. J Pharmacol Exp Ther 311:864-873

58. van der Leij FR, Bloks VW, Grefhorst A et al (2007) Gene expression profiling in livers of mice after acute inhibition of beta-oxidation. Genomics 90:680-689

59. Fromenty B (2013) Drug-induced liver injury in obesity. J Hepatol 58:824-826

60. Lettéron P, Sutton A, Mansouri A et al (2003) Inhibition of microsomal triglyceride transfer protein: another mechanism for drug-induced steatosis in mice. Hepatology 38:133-140

61. - Anthérieu S, Rogue A, Fromenty B, et al (2011) Induction of vesicular steatosis by amiodarone and tetracycline is associated with up-regulation of lipogenic genes in HepaRG cells. Hepatology 53:1895-1905. This article was the first report demonstrating that amiodarone and tetracycline was able to induce vesicular steatosis in the well-differentiated human hepatoma HepaRG cells. This study confirmed that both drugs can inhibit mitochondrial $\beta$-oxidation as previously described [21, 37, 4244, 74-76], but also revealed that amiodarone could also increase de novo lipogenesis.

62. Genève J, Hayat-Bonan B, Labbe G et al (1987) Inhibition of mitochondrial $\beta$-oxidation of fatty acids by pirprofen. Role in microvesicular steatosis due to this nonsteroidal anti-inflammatory drug. J Pharmacol Exp Ther 242:1133-1137

63. Bickel PE, Tansey JT, Welte MA (2009) PAT proteins, an ancient family of lipid droplet proteins that regulate cellular lipid stores. Biochem Biophys Acta 1791:419-440

64. Scaini G, Simon KR, Tonin AM et al (2012) Toxicity of octanoate and decanoate in rat peripheral tissues: evidence of bioenergetic dysfunction and oxidative damage induction in liver and skeletal muscle. Mol Cell Biochem 361:329-335

65. Kleiner DE, Gaffey MJ, Sallie R et al (1997) Histopathologic changes associated with fialuridine hepatotoxicity. Mod Pathol 10:192-199

66. Caldwell SH, Hespenheide EE, von Borstel RW (2001) Myositis, microvesicular hepatitis, and progression to cirrhosis from troglitazone added to simvastatin. Dig Dis Sci 46:376-378

67. Fukano M, Amano S, Sato J et al (2000) Subacute hepatic failure associated with a new antidiabetic agent, troglitazone: a case report with autopsy examination. Hum Pathol 31:250-253

68. Kohlroser J, Mathai J, Reichheld J et al (2000) Hepatotoxicity due to troglitazone: report of two cases and review of adverse events reported to the United States Food and Drug Administration. Am J Gastroenterol 95:272-276

69. Derks TG, van Dijk TH, Grefhorst A et al (2008) Inhibition of mitochondrial fatty acid oxidation in vivo only slightly suppresses gluconeogenesis but enhances clearance of glucose in mice. Hepatology 47:1032-1042

70. Le Dinh T, Freneaux E, Labbe G et al (1988) Amineptine, a tricyclic antidepressant, inhibits the mitochondrial oxidation of fatty acids and produces microvesicular steatosis of the liver in mice. J Pharmacol Exp Ther 247:745-750

71. - Shi X, Yao D, Gosnell BA, et al (2012) Lipidomic profiling reveals protective function of fatty acid oxidation in cocaineinduced hepatotoxicity. J Lipid Res. 53:2318-2330. This study epitomizes the fact that non-pharmaceutical compounds are able to inhibit mitochondrial $\beta$-oxidation and cause microvesicular steatosis. In this study, cocaine-induced inhibition of hepatic $\beta$ oxidation was associated with the accumulation of palmitoylcarnitine in serum and liver. Interestingly, accretion of acylcarnitine derivatives can be also observed with valproic acid.

72. Fu Q, Huang X, Shu B et al (2011) Inhibition of mitochondrial respiratory chain is involved in triptolide-induced liver injury. Fitoterapia 82:1241-1248

73. Wan HT, Zhao YG, Wei X et al (2012) PFOS-induced hepatic steatosis, the mechanistic actions on $\beta$-oxidation and lipid transport. Biochim Biophys Acta 1820:1092-1101

74. Spaniol M, Bracher R, Ha HR et al (2001) Toxicity of amiodarone and amiodarone analogues on isolated rat liver mitochondria. J Hepatol 35:628-636

75. Kaufmann P, Török M, Hänni A et al (2005) Mechanisms of benzarone and benzbromarone-induced hepatic toxicity. Hepatology 41:925-935

76. • Felser A, Blum K, Lindinger PW, et al (2013) Mechanisms of hepatocellular toxicity associated with dronedarone. A comparison to amiodarone. Toxicol Sci. 131:480-490. This article showed for the first time that the new antiarrhythmic drug dronedarone is able to impair mitochondrial function in a similar way that amiodarone, its structural analogue. Moreover, this article and others (see for instance [74] and [75]) confirmed our original data published in 1990 showing that amiodarone can have a dual effect on OXPHOS (i.e. uncoupling and inhibition) and inhibit mitochondrial fatty acid $\beta$-oxidation $[11,21]$.

77. Surendradoss J, Chang TK, Abbott FS (2012) Assessment of the role of in situ generated (E)-2,4-diene-valproic acid in the toxicity of valproic acid and (E)-2-ene-valproic acid in sandwichcultured rat hepatocytes. Toxicol Appl Pharmacol 264:413-422

78. Walker UA, Bäuerle J, Laguno M et al (2004) Depletion of mitochondrial DNA in liver under antiretroviral therapy with didanosine, stavudine, or zalcitabine. Hepatology 39:311-317

79. Boubaker K, Flepp M, Sudre P et al (2001) Hyperlactatemia and antiretroviral therapy: the Swiss HIV Cohort Study. Clin Infect Dis 33:1931-1937

80. Finsterer J, Segall L (2010) Drugs interfering with mitochondrial disorders. Drug Chem Toxicol 33:138-151

81. Bailey CM, Kasiviswanathan R, Copeland WC et al (2009) R964C mutation of DNA polymerase $\gamma$ imparts increased stavudine toxicity by decreasing nucleoside analog discrimination and impairing polymerase activity. Antimicrob Agents Chemother 53:2610-2612

82. - Stewart JD, Horvath R, Baruffini E, et al (2010) Polymerase $\gamma$ gene POLG determines the risk of sodium valproate-induced liver toxicity. Hepatology. 52:1791-1796. This article showed for the first time that a particular genetic variation in the POLG gene was strongly associated with valproate-induced liver toxicity. This gene is encoding the DNA polymerase $\gamma$, which is the only DNA polymerase involved in mtDNA replication. However, it is still unclear how mutations in the POLG gene could favor VPA-induced liver injury, contrary to antiretroviral nucleoside analogue-induced toxicity [81].

83. Degoul F, Sutton A, Mansouri A et al (2001) Homozygosity for alanine in the mitochondrial targeting sequence of superoxide dismutase and risk for severe alcoholic liver disease. Gastroenterology 120:1468-1474

84. Boelsterli UA, Hsiao CJ (2008) The heterozygous Sod $2^{+/-}$ mouse: modeling the mitochondrial role in drug toxicity. Drug Discov Today 13:982-988

85. Begriche K, Lettéron P, Abbey-Toby A et al (2008) Partial leptin deficiency favors diet-induced obesity and related metabolic disorders in mice. Am $\mathrm{J}$ Physiol Endocrinol Metab 294:E939-E951 
86. Farrell GC (2002) Drugs and steatohepatitis. Semin Liver Dis 22:185-194

87. Grismer LE, Gill SA, Harris MD (2001) Liver biopsy in psoriatic arthritis to detect methotrexate hepatotoxicity. J Clin Rheumatol 7:224-227

88. Zorzi D, Laurent A, Pawlik TM et al (2007) Chemotherapyassociated hepatotoxicity and surgery for colorectal liver metastases. Br J Surg 94:274-286

89. Berson A, De Beco V, Lettéron P et al (1998) Steatohepatitisinducing drugs cause mitochondrial dysfunction and lipid peroxidation in rat hepatocytes. Gastroenterology 114:764-774

90. Mitchell C, Mahrouf-Yorgov M, Mayeuf A et al (2011) Overexpression of $\mathrm{Bcl}-2$ in hepatocytes protects against injury but does not attenuate fibrosis in a mouse model of chronic cholestatic liver disease. Lab Invest 91:273-282

91. Brady LJ, Brady PS, Romsos DR et al (1985) Elevated hepatic mitochondrial and peroxisomal oxidative capacities in fed and starved adult obese (ob/ob) mice. Biochem J 231:439-444

92. Takamura T, Shimizu A, Komura T et al (2007) Selective estrogen receptor modulator raloxifene-associated aggravation of nonalcoholic steatohepatitis. Intern Med 46:579-581

93. Igoudjil A, Massart J, Begriche K et al (2008) High concentrations of stavudine impair fatty acid oxidation without depleting mitochondrial DNA in cultured rat hepatocytes. Toxicol In Vitro 22:887-898

94. Martins-Maciel ER, Campos LB, Salgueiro-Pagadigorria CL et al (2013) Raloxifene affects fatty acid oxidation in livers from ovariectomized rats by acting as a pro-oxidant agent. Toxicol Lett 217:82-89
95. Nazarewicz RR, Zenebe WJ, Parihar A et al (2007) Tamoxifen induces oxidative stress and mitochondrial apoptosis via stimulating mitochondrial nitric oxide synthase. Cancer Res 67:1282-1290

96. Singh K, Malviya A, Bhori M et al (2012) An in vitro study of the ameliorative role of $\alpha$-tocopherol on methotrexate-induced oxidative stress in rat heart mitochondria. J Basic Clin Physiol Pharmacol 23:163-168

97. Cole LK, Jacobs RL, Vance DE (2010) Tamoxifen induces triacylglycerol accumulation in the mouse liver by activation of fatty acid synthesis. Hepatology 52:1258-1265

98. • Hynes J, Swiss RL, Will Y (2012) High-throughput analysis of mitochondrial oxygen consumption. Methods Mol Biol. 810:59-72. This article provides detailed protocols for the measurement of mitochondrial oxygen consumption with the phosphorescent water-soluble oxygen probe MitoXpress ${ }^{\circledR}$. This methodology can allow the high-throughput assessment of druginduced alteration of mitochondrial function such as MRC activity and mtFAO ([12] and Table 2).

99. Robinson SM, Wilson CH, Burt AD et al (2012) Chemotherapyassociated liver injury in patients with colorectal liver metastases: a systematic review and meta-analysis. Ann Surg Oncol 19:4287-4299

100. Wang Y, Lin Z, Liu Z et al (2013) A unifying ontology to integrate histological and clinical observations for drug-induced liver injury. Am J Pathol 182:1180-1187 